\title{
BMPER Enhances Bone Formation by Promoting the Osteogenesis-Angiogenesis Coupling Process in Mesenchymal Stem Cells
}

\author{
Fei Xiao Chuandong Wang Chenglong Wang Yuan Gao Xiaoling Zhang \\ Xiaodong Chen \\ Department of Orthopedic Surgery, Xin Hua Hospital Affilliated to Shanghai Jiao Tong University \\ School of Medicine (SJTUSM), Shanghai, China
}

\section{Key Words}

Bmper • Osteogenic differentiation • Angiogenesis - Mesenchymal Stem Cells

\begin{abstract}
Background/Aims: During bone repair and remodeling, osteogenesis is coupled with angiogenesis. Bone morphogenetic protein (BMP) antagonists are important modulators of BMP signaling and bone homeostasis. Several investigations have demonstrated that one 'BMP antagonist', BMP-binding endothelial cell precursor-derived regulator (BMPER), participates in the regulation of BMP signaling. In this study, we examined the role of BMPER in the osteogenesis-angiogenesis coupling process. Methods: Human bone mesenchymal stem cells (hBMSCs) and human umbilical vein endothelial cells (HUVECs) were used in this experiment. After overexpressing or silencing BMPER with lentiviruses or siRNA, hBMSCs were stimulated by BMP-2, and osteogenic differentiation activity was detected by alkaline phosphatase and alizarin red staining. VEGF and endostatin release were assessed by ELISA. HUVEC migration was detected by the cell scratch test and transwell migration assay, and in vitro angiogenesis was determined by the tube formation assay. Bone formation was assessed using in vivo femoral monocortical defect and ectopic bone formation models. Results: BMP2 upregulated BMPER expression. Overexpression of BMPER remarkably enhanced BMP-2induced osteogenic differentiation, while suppression of $B M P E R$ effectively inhibited this process both in vitro and in vivo. In addition, overexpression of BMPER promoted BMP-2induced VEGF expression in vitro and vascularization in the ectopic bone formation model. Conclusion: BMPER functions as a positive regulator of the osteogenesis-angiogenesis coupling process in $\mathrm{hBMSCs}$, suggesting a novel therapeutic role of BMPER in the regenerative capacity of bone repair.
\end{abstract}

F. Xiao, CD. Wang and CL. Wang contributed to the work equally.

Xiaoling Zhang and Xiaodong Chen 


\section{Cellular Physiology Cell Physiol Biochem 2018;45:1927-1939 \\ and Biochemistry Published online: March 08, $2018 \quad \begin{aligned} & \text { DOI: 10.1159/000487969 } 2018 \text { The Author(s). Published by S. Karger AG, Basel } \\ & \text { www.karger.com/cpb }\end{aligned}$ \\ Xiao et al.: BMPER Enhances Bone Formation}

\section{Introduction}

Bone repair following fracture is usually a rapid and precise process. After a fracture, bone mesenchymal stem cells (BMSCs) migrate to the injured site and gradually differentiate into osteoblasts, which produce bone matrix and repair the fractured bone [1]. While multiple signaling pathways are involved in regulating BMSC differentiation, bone morphogenetic proteins (BMPs) play a critical role in inducing the differentiation of BMSCs of the osteoblastic lineage and enhancing the differentiated function of osteoblasts [2, 3]. BMP-2, BMP-4, BMP6, BMP-7 and BMP-9 induce osteogenic differentiation of MSCs in vitro and in vivo $[4,5]$. By binding type I and II receptors to form complexes on the cell surface, BMPs exert their biological function through the activation of Smad signaling pathways, which regulate osteogenic-related gene expression [6,7]. Meanwhile, BMPs are functionally modulated by a class of intracellular and extracellular BMP-binding proteins, termed BMP antagonists $[2,8]$. To date, noggin, gremlin, chordin, follistatin, and sclerostin have been identified as osteoblast products that bind BMPs $[8,9]$. Some evidence exists that BMP antagonists, such as gremlin-1 and gremlin-2, limit BMP activity during osteogenesis, and the BMP-2induced osteogenesis of BMSCs can be markedly enhanced by suppression of gremlin- 1 and gremlin-2 expression $[4,5]$. However, some 'BMP antagonists', such as noggin, play varying roles during osteogenesis in different species. Noggin inhibits osteogenesis by preventing BMPs from binding their receptors on the cell surface in some animal models, such as mice $[10,11]$, and enhances osteogenesis by inducing BMP-2 and osteocalcin in human BMSCs (hBMSCs) $[1,12]$.

Another 'BMP antagonist', BMP-binding endothelial cell precursor-derived regulator (BMPER;also known as Crossveinless 2 [CV2]) exerts both stimulatory and antagonistic effects on BMP actions in different contexts $[13,14]$. BMPER binds and modulates at least three BMPs (BMP-2, BMP-4 and BMP-6) and was originally discovered in a screen for differentially expressed proteins in Drosophila embryonic endothelial precursor cells [15]. Loss-of-function models demonstrated that BMPER acts as a pro-BMP modulator most likely by facilitating the binding of BMPs to their respective receptors $[13,16]$. A number of studies have clarified the function of BMPER in the vasculature, and research focused on the skeletal system is increasing. Bmper knockout mice show defects in vertebral and cartilage development and renal hypoplasia [17]. Furthermore, mutations in the BMPER gene have been reported in diaphanospondylodysostosis and ischiospinal dysostyosis, both of which result in the skeletal dysostosis phenotype $[18,19]$. As a regulator of the BMP signaling pathway, BMPER plays a critical role in vertebral and cartilage development. However, the role of $B M P E R$ in hBMSC differentiation is still poorly understood.

Angiogenesis and osteogenesis are two intimately, well-coordinated coupling processes during bone healing [20]. Interactions between MSCs and endothelial cells (ECs) are mediated by numerous angiogenic factors, growth factors and cytokines and have been discovered in the MSC secretome [21]. Conversely, blood vessels contribute to the process of osteogenesis during bone repair [22, 23]. In addition, these processes are also very important for the regulation of endochondral bone growth. Vascular invasion of the primarily avascular hypertrophic zone of the growth plate brings osteoblasts and endothelial precursor cells into future centers of ossification [24]. Patients with skeletal dysplasia ischiospinal dysostosis caused by inactivated $B M P E R$ mutations suffer not only from vertebral and rib malformations but also from significantly short statures [18]. Furthermore, the lack of Bmper translates into apparent endothelial thickening, and an increasing number of immature ECs have been observed in Bmper-deficient mice [16, 25]. Thus, we hypothesize that BMPER may be involved in the angiogenesis and osteogenesis processes and even in the coupling process.

In the present study, BMP-2 upregulates BMPER expression during hBMSC osteogenic differentiation. Overexpression of $B M P E R$ by lentiviruses enhances the osteogenic capability of hBMSCs, while suppression of BMPER exerts the opposite effect. Furthermore, BMPER accelerates bone formation by promoting the osteogenesis-angiogenesis coupling process in ectopic osteogenesis and monocortical defect models. Thus, we provide novel mechanistic 


\section{Cellular Physiology Cell Physiol Biochem 2018;45:1927-1939 \\ \begin{tabular}{l|l} 
and Biochemistry Published online: March 08, 2018 & $\begin{array}{l}\text { DOI: } 2018 \text { The Author(s). Published by S. Karger AG, Basel } \\
\text { www.karger.com/cpb }\end{array}$
\end{tabular}}

Xiao et al.: BMPER Enhances Bone Formation

insights into hBMSC osteogenesis and show that BMPER is a potential therapeutic target for bone repair in clinical practice.

\section{Materials and Methods}

\section{Isolation and expansion of $h B M S C s$}

This experiment was approved by the Institutional Ethics Committee of Xin Hua Hospital Affiliated to Shanghai Jiao Tong University School of Medicine. hBMSCs were donated by 3 male and 3 female patients aged 40.7 years (range, 35-47 years) who accepted traumatic femoral shaft fracture treatment by intramedullary nailing. Informed consent was obtained from all donors. None of the patients presented with osteoporosis, other orthopedic diseases or systemic diseases. Bone marrow blood aspirated during reaming was filtered through a 100- $\mu \mathrm{m}$ nylon mesh cell strainer to remove trabecular bone debris. The filtrate $(2$ $\mathrm{mL}$ ) from the femurs of each donor was inoculated in a $10-\mathrm{cm}$ dish containing basal medium (BM; $\alpha$-MEM [HyClone, USA] supplemented with $10 \%$ fetal bovine serum [FBS;Gibco, USA], $100 \mathrm{U} / \mathrm{mL}$ penicillin G, and $100 \mathrm{mg} / \mathrm{L}$ streptomycin [HyClone]) at $37^{\circ} \mathrm{C}$ in a humidified atmosphere containing $5 \% \mathrm{CO}_{2}$. After 3 days in culture, non-adherent cells were removed from the culture supernatant. hBMSCs that adhered to the dish were cultured in complete medium that was replaced every 2 days. When the cells reached confluence, they were detached using $0.25 \%$ trypsin (Gibco) and then stored or reseeded for culture. Cells were used for subsequent experiments between passages 2 and 5 .

\section{Identification of the hBMSC lineage}

hBMSCs $\left(3 \times 10^{5}\right.$ cells) at passage 3 were incubated with fluorescein-conjugated mouse anti-human monoclonal antibodies for $45 \mathrm{~min}$ at room temperature. After the cells were washed with fluorescenceactivated cell sorting (FACS) buffer (phosphate buffered saline [PBS] with 10\% bovine serum albumin [BSA] and $1 \%$ sodium azide) at $300 \mathrm{~g}$ for $5 \mathrm{~min}$, the stained cells were resuspended in $250 \mu \mathrm{L}$ of ice-cold FACS buffer and then subjected to FACS analysis (BD Biosciences, USA). A total of $1 \times 10^{4}$ events were counted for each sample. The percentage of cells showing a positive signal was analyzed using FlowJo software (Tree Star, USA). The antibodies, including anti-CD34, anti-CD45, anti-CD90, anti-CD29, anti-CD105 and antiCD44, were purchased from BD Biosciences.

\section{Multi-lineage differential potential}

For osteogenic differentiation assays, hBMSCs at passage 3 were seeded at $1 \times 10^{5}$ cells/well in a sixwell culture plate and cultured in BM until confluent. Then, the cells were cultured in BM or osteogenic medium (OM;BM supplemented with $100 \mathrm{ng} / \mathrm{mL}$ BMP-2 [PeproTech, USA]) for 21 days for the assessment of alizarin red staining. For chondrogenic differentiation assays, hBMSCs at passage 3 were resuspended in $\mathrm{BM}$ at a density of $1 \times 10^{7}$ cells $/ \mathrm{mL}$. Three drops containing a $10-\mu \mathrm{L}$ cell suspension were carefully added to a 12-well plate. The cells were allowed to adhere at $37^{\circ} \mathrm{C}$ in $5 \% \mathrm{CO}_{2}$ for $2 \mathrm{~h}$, followed by the addition of $1 \mathrm{ml}$ of BM or chondrogenic medium (CM;BM supplemented with $10 \mathrm{ng} / \mathrm{mL}$ recombination human transforming growth factor-beta 1 [rhTGF- $\beta 1$;PeproTech], $1 \times$ ITS [Invitrogen, USA], $0.5 \mathrm{mg} / \mathrm{mL}$ BSA, $4.7 \mu \mathrm{g} / \mathrm{mL}$ linoleic acid, $50 \mu \mathrm{g} / \mathrm{ml}$ L-proline, $100 \mathrm{nM}$ dexamethasone, and $37.5 \mu \mathrm{g} / \mathrm{mL}$ ascorbic acid). The culture medium was changed every 3 days. Micromasses were fixed for paraffin sectioning and alcian blue staining after 21 days. For adipogenic differentiation assays, hBMSCs at passage 3 were seeded at $1 \times 10^{5}$ cells/well in a six-well plate and then cultured with BM. When confluence was reached, the cells were cultured in BM or adipogenic medium (AM;BM supplemented with $500 \mathrm{nM}$ dexamethasone, $0.5 \mathrm{mM}$ isobutylmethylxanthine, $50 \mathrm{mM}$ indomethacin, and $10 \mathrm{mg} / \mathrm{mL}$ insulin). The medium was changed every 3 days. After 14 days in adipogenic culture, the cells were fixed for oil red $O$ staining. Unless otherwise indicated, all other reagents were purchased from Sigma-Aldrich (St. Louis, USA).

\section{Lentiviral transduction overexpression study}

Human BMPER genes were ligated into the pLVX-IRES-Puro vector using PCR primers to amplify the coding region. The pLVX-IRES-Puro and pLVX-IRES-Puro-BMPER constructs were transfected into the HEK293T viral packaging cell line together with pSPAX2 and pMD2.G. Viral supernatant was used for the infection of hBMSCs. 


\section{Cellular Physiology Cell Physiol Biochem 2018;45:1927-1939 \begin{tabular}{l|l|l}
\hline DOI: 10.1159/000487969 & $\begin{array}{l}\text { C) } 2018 \text { The Author(s). Published by S. Karger AG, Basel } \\
\text { www.karger.com/cpb }\end{array}$
\end{tabular}}

Xiao et al.: BMPER Enhances Bone Formation

SiRNA transfection

siRNA transfection was carried out using Lipofectamine 2000 (Invitrogen) according to the manufacturer's instructions. The sequences of siRNA targeting BMPER (GenePharma Co., Ltd., China) were as follows:

siRNA1: sense 5'-GCGCUGUGUUGUUCAUUGUTT dTdT-3' and antisense 5'-ACAAUGAACAACACAGCGCTT dTdT-3';

siRNA2: sense 5'-GACGGUCGGACAUUUAACUTT dTdT-3' and antisense 5'-AGUUAAAUGUCCGACCGUCTT dTdT-3';and

siRNA3: sense 5'-GCAACUACAAUGGACAUAATT dTdT-3' and antisense 5'-UUAUGUCCAUUGUAGUUGCTT dTdT-3'.

The sequences of BMPER negative control siRNA (BMPER NC siRNA) were as follows: sense 5'UUCUCCGAACGUGUCACGUTT dTdT-3' and antisense 5'-ACGUGACACGUUCGGAGAATT dTdT-3'.

\section{Real-time PCR analysis}

Total RNA was extracted using TRIzol reagent and reverse transcribed using the PrimeScript RT Master Mix cDNA Synthesis Kit (Takara, Japan) to obtain first strand cDNA. Real-time PCR reactions were performed on the VIIA7 instrument (Applied Biosystems, USA) using the SYBR Green PCR Kit (TaKaRa). The real-time PCR conditions were set as follows: denaturation at $95{ }^{\circ} \mathrm{C}$ for $30 \mathrm{~s}$, followed by 50 cycles of $95{ }^{\circ} \mathrm{C}$ for $10 \mathrm{~s}$ and $60{ }^{\circ} \mathrm{C}$ for $30 \mathrm{~s}$. All quantitation was normalized to an endogenous control, GAPDH, and data were analyzed using the $2^{-\Delta \Delta C \mathrm{~T}}$ method. The sequences of the gene primers used are listed in Table 1. All experiments were repeated independently in triplicate.

\section{Western blot analysis}

Total proteins were extracted from hBMSCs using RIPA buffer (Beyotime), and the protein concentrations were determined using a BCA protein assay (Thermo Fisher). Lysate proteins (30 $\mu \mathrm{g}$ ) were separated by $10 \%$ SDS-PAGE and transferred to polyvinylidene difluoride membranes. The membranes were then blocked with 5\% skim milk in TBS-Tween (TBS: $0.05 \mathrm{M}$ Tris and $0.15 \mathrm{M} \mathrm{NaCl}$, pH 7.4;0.2\% Tween-20) for 1 $\mathrm{h}$ and incubated with primary antibodies diluted in $1 \%(\mathrm{w} / \mathrm{v})$ skim milk powder in TBS-Tween overnight at $4{ }^{\circ} \mathrm{C}$. A horseradish peroxidase-labeled secondary antibody was added and visualized using an enhanced chemiluminescence detection system (Millipore, USA) in accordance with the manufacturer's instructions. All experiments were repeated independently in triplicate.

\section{Osteogenic differentiation assay}

The cells were washed twice with PBS, fixed in 4\% polyoxymethylene for $15 \mathrm{~min}$ and stained with alkaline phosphatase reagent (ALP;Shanghai Hongqiao Le Xiang Institute of Biomedical) or alizarin red (Sigma) staining solution at $37^{\circ} \mathrm{C}$ for $30 \mathrm{~min}$. After three washes with distilled water, the stained cells were photographed. For quantitative ALP measurements, the cells were rinsed with PBS in triplicate and lysed with RIPA lysis buffer. Next, the cell supernatants were collected in 96-well plates. Substrates and p-nitrophenol from the Alkaline Phosphatase Assay Kit (Beyotime) were added to the 96-well plates, and ALP activity was determined at a wavelength of $405 \mathrm{~nm}$ after a 30 -min incubation at $37^{\circ} \mathrm{C}$. Finally, the ALP levels were normalized to the total protein content determined by the Bicinchoninic Acid Protein Assay Kit (Beyotime). For the quantification of calcium deposits, $0.1 \mathrm{~N} \mathrm{NaOH}$ was added to dissolve extracellular calcium deposits, and $100 \mu \mathrm{L}$ of the dissolved solution was detected using a microplate reader (TECAN, Switzerland) at $548 \mathrm{~nm}$. All experiments were repeated independently in triplicate.

Table 1. List of primers used for real-time PCR analysis

\begin{tabular}{lcc}
\hline Gene name & Forward primer(5'-3') & Reverse primer(5'-3') \\
\hline CHORDIN & TTCGGCGGGAAGGTCTATG & ACTCTGGTTTGATGTTCTTGCAG \\
TWSG1 & ATTGGAACATCGTTTCTTTCCCT & ACCAGCGATATTTGGATGCTC \\
BMPER & TTTATCACAGACAACCCTTGCAT & TCCTGGCACTGGCGTAGAA \\
NOGGIN & CCATGCCGAGCGAGATCAAA & TCGGAAATGATGGGGTACTGG \\
GREM1 & CGGAGCGCAAATACCTGAAG & GGTTGATGATGGTGCGACTGT \\
SOST & ACACAGCCTTCCGTGTAGTG & GGTTCATGGTCTTGTTGTTCTCC \\
CERBERUS & TCCAGGGACTCAGATAGTGAGC & GCAGGTCTCCCAATGTACTTCA \\
USAG1 & AACAGCACGTTGAATCAAGCC & GCCATCAGAGATGTATTTGGTGG \\
COCO & AAGTGATCCAGGGGATGTGTA & GATGATTTCGGAGGCGTATGG \\
PRDC & ATCCCCTCGCCTTACAAGGA & TCTTGCACCAGTCACTCTTGA \\
DAN & CATGTGGGAGATTGTGACGCT & CCTCGTGACTAGGCTCCTTG \\
COL1A1 & GAGGGCCAAGACGAAGACATC & CAGATCACGTCATCGCACAAC \\
OCN & GAAGCCCAGCGGTGCA & CACTACCTCGCTGCCCTCC \\
OSX & CCTCTGCGGGACTCAACAAC & AGCCCATTAGTGCTTGTAAAGG \\
GAPDH & ACAACTTTGGTATCGTGGAAGG & GCCATCACGCCACAGTTTC \\
\hline
\end{tabular}




\section{Cellular Physiology Cell Physiol Biochem 2018;45:1927-1939 \begin{tabular}{c|c|c|} 
DOI: 10.1159/000487969 & Cand Biochemistry & $\begin{array}{l}\text { O } 2018 \text { The Author(s). Published by S. Karger AG, Basel } \\
\text { www.karger.com/cpb }\end{array}$
\end{tabular} \\ Xiao et al.: BMPER Enhances Bone Formation}

ELISA

hBMSCs were seeded on a six-well plate at a density of $1 \times 10^{5}$ cells/well and induced by OM after lentiviral infection or siRNA transfection. The medium was changed twice a week, and the conditional medium was collected at the second medium change for the following experiments. The concentrations of VEGF and endostatin in the conditional medium were measured using an ELISA kit (Thermo Fisher, USA) according to the manufacturer's instructions. All experiments were repeated independently in triplicate.

\section{Cell scratch test}

HUVECs were seeded $\left(1 \times 10^{5}\right.$ cells/well) in $1 \%$ gelatin-coated 24 -well plates (Corning, Netherlands). Confluent cells were serum-deprived for $16 \mathrm{~h}$, and linear wounds were created in the monolayers by scratching with a sterile pipette tip. Next, the monolayers were washed with PBS to remove floating cells, and the conditional medium was added. After an additional $24 \mathrm{~h}$, cell migration into the wound was assessed by microscopy. All experiments were repeated independently in triplicate.

\section{Transwell migration assay}

A transwell migration assay was performed using Transwell inserts (BD Biosciences) with an 8- $\mu \mathrm{m}$ pore size filter. A total of $5 \times 10^{4}$ cells were seeded in serum-free medium into the upper chamber of the insert precoated with Matrigel, and $700 \mu \mathrm{l}$ of conditional medium was added to the lower chamber. After $24 \mathrm{~h}$ of incubation, the cells were fixed with $75 \%$ ethanol and stained with crystal violet. Then, the cells on the top surface of the membrane were carefully wiped off, and the cells on the lower surface were examined with a microscope. Five random fields were photographed for counting purposes, and the average number of migrated cells was used as a measure of migration capacity. All experiments were repeated independently in triplicate.

\section{Tube formation assay}

HUVECs were serum starved for $16 \mathrm{~h}$ and then seeded at a density of $4 \times 10^{4}$ per well on growth factordepleted Matrigel (BD Biosciences) in 48-well plates. Conditional medium was added, and the results were quantified after $4 \mathrm{~h}$. Microscopic fields containing the tube structures formed in the gel were photographed at low magnification $(\times 10)$. Five fields per test condition were examined. Before being photographed, the cells were fixed with $4 \%$ paraformaldehyde. All experiments were repeated independently in triplicate.

\section{Immunofluorescence assay}

Immunostaining was performed using a standard protocol. After stimulation with BMP-2 for 7 days, hBMSCs were incubated with the following primary antibodies: BMPER rabbit pAb (Abcam, USA), VEGF mouse pAb (Abcam) and COL1A1 mouse pAb (Abcam) overnight at $4{ }^{\circ} \mathrm{C}$. Then, the primary antibodies were detected using Cy3- or FITC-conjugated anti-mouse or rabbit IgG secondary antibodies (Life Technologies, USA). After the final wash, nuclei were counterstained with Hoechst 3342 (Life Technologies) in PBS for 5 min before imaging. The stained sections were immediately observed by laser confocal microscopy (ZEISS, Germany). All experiments were repeated independently in triplicate.

\section{Ectopic bone formation assay}

This animal experiment was approved by the Institutional Ethics Committee of Xin Hua Hospital Affiliated to Shanghai Jiao Tong University School of Medicine. Subcutaneous stem cell implantation was performed as previously described [26, 27]. Briefly, BMSCs were infected with different lentiviruses. The cells were collected $24 \mathrm{~h}$ after infection, and approximately $3.0 \times 10^{6}$ cells were mixed with $\beta$-TCP ceramic particles (50 mg, Shanghai Bio-lu Biomaterials Co., Ltd, China). This mixture was subcutaneously implanted into the dorsal surfaces of nude mice. After 6 or 12 weeks, the implants were harvested, fixed in $4 \%$ paraformaldehyde, decalcified with an ultrasonic decalcification instrument, and embedded in paraffin. For histological analyses, the sections $(7 \mu \mathrm{m})$ were stained with hematoxylin and eosin (HE) and Masson's Trichrome stain.

Immunohistochemical staining

Sections were deparaffinized and rehydrated. Antigen retrieval was performed by digestion with pepsin (Sigma) at $37^{\circ} \mathrm{C}$ for several minutes. The sections were blocked with $5 \%$ goat serum and $0.1 \%$ 


\section{Cellular Physiology Cell Physiol Biochem 2018;45:1927-1939

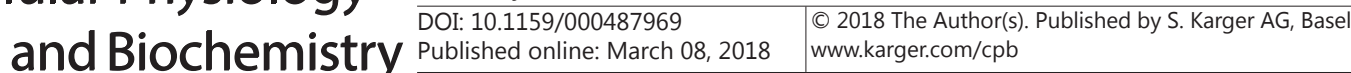 \\ Xiao et al.: BMPER Enhances Bone Formation}

Tween-20 in PBS for 30 min at room temperature and then incubated with an anti-CD31 primary antibody (Abcam) overnight at $4{ }^{\circ} \mathrm{C}$. A streptavidin-horse radish peroxidase (HRP) detection system was used to detect the antigens, which were visualized with 2,2 -diaminobenzidine tetrahydrochloride. The sections were then counterstained with hematoxylin and sealed with mounting medium.

\section{Femoral monocortical defect model}

This animal experiment was approved by the Institutional Ethics Committee of Xin Hua Hospital Affiliated to Shanghai Jiao Tong University School of Medicine. The femoral monocortical defect model was established as previously described $[4,28]$. Briefly, nude mice were placed under general anesthesia, and the lateral aspects of their right femurs were exposed. The overlying soft tissues were pushed aside, and the periosteum was carefully preserved. A monocortical osseous hole $(0.8 \mathrm{~mm}$ diameter $)$ was created on the femur lateral surface using a round burr attached to a dental drill. Irrigation with saline was used to remove bone dust and fragments. Approximately $5.0 \times 10^{4} \mathrm{hBMSC}$ were exposed to different treatments for $24 \mathrm{~h}$, resuspended in a mixture of medium and Matrigel, and then transplanted into the osseous hole. After one month, the right femurs were fixed in $4 \%$ paraformaldehyde for $24 \mathrm{~h}$ at $4{ }^{\circ} \mathrm{C}$. The samples were scanned using high-resolution microcomputed tomography ( $\mu$ CT) (SCANCO, Switzerland) with a spatial resolution of $5 \mu \mathrm{m}$. Sagittal image sections of the model femurs were used to perform 3D histomorphometric analysis. We defined the regions of interest as (i) the hole region between the interrupted cortical bone ends, (ii) injured bone marrow, and (iii) periosteal callus outside the hole. Old bone fragments remaining from the drilling were excluded from the regions of interest. A total of 100 consecutive images were used for 3D reconstruction and analysis, covering most of the injured region and periosteal callus. Structural parameters included bone mineral density (BMD) and bone volume percentage (bone volume/total volume, BV/TV). Fixed samples were decalcified and embedded in paraffin for HE staining.

\section{Statistical analysis}

Statistical significance was assessed using two-tailed Student's t-tests or analysis of variance (ANOVA). All statistical analyses were performed using SPSS software version 19.0. Statistical significance was considered at $\mathrm{P}<0.05\left(^{*}\right)$ and $\mathrm{P}<0.01\left(^{* *}\right)$. Data are presented as the means \pm S.Ds.

\section{Results}

\section{Identification and characterization of hBMSCS}

Isolated hBMSCs expressed the MSC markers CD29, CD44, CD90, and CD105 and did not express the hematopoietic marker CD34 or the leukocyte marker CD45 (Fig. 1A). The adipogenic differentiation capacity of hBMSCs was verified by oil red 0 staining. Lipid droplets were formed in hBMSCs after 14 days of adipogenic induction but were not observed in hBMSCs cultured in BM (Fig. 1B). The osteogenic differentiation assay indicated that most cells formed mineralized calcium deposits, as confirmed by alizarin red staining after 21 days of induction (Fig. 1C). Compared with chondrogenic induction in BM, chondrogenic induction in CM resulted in higher alcian blue staining in the hBMSC micromass after 21 days (Fig. 1D).

\section{Expression of BMPER during the osteogenic differentiation of hBMSCs}

The mRNA expression levels of all the involved BMP inhibitors showed varying degrees of change during osteogenic differentiation, and BMPER expression increased over time except for a decrease on day 21 compared with that on day 14 (Fig. 2A). Western blot analysis showed that BMPER was significantly upregulated over time, with an approximately 5-fold increase in protein levels after 21 days in the osteogenic induction group compared with those in the non-induction group (Fig. 2B and C).

BMPER positively regulates osteogenic differentiation in hBMSCs

To confirm the effect of BMPER on hBMSC osteogenesis, we used lentiviruses expressing $B M P E R$ and siRNA targeting BMPER. Three siRNAs were synthesized, and while they all 
showed inhibition effects (data not shown), only SiRNA 1 was used in the subsequent experiment because it exhibited the highest inhibition efficiency. The levels of BMPER mRNA and protein expression were significantly increased by pLVX-BMPER (Fig. 3A and $B$ ) and suppressed by siRNA 1 (Fig. 3C and D). The ALP assay showed that pLVX-BMPER infection increased the ALP activity in the cells after 7 days of osteogenic induction, while transfection of the siRNA exerted the opposite effect (Fig. 3E and G). Qualitative alizarin red staining and the quantitative calcium assay showed that more calcium deposits were produced by the BMPER overexpression group than by the negative control (NC) and pLVXvector groups after 21 days of osteogenic induction (Fig. $3 \mathrm{~F}$ and $\mathrm{H}$ ).

After 7 days of osteogenic induction, the mRNA expression levels of osteogenesis-related genes, including $O S X, O C N$, and COL1A1, in the pLVX-BMPER

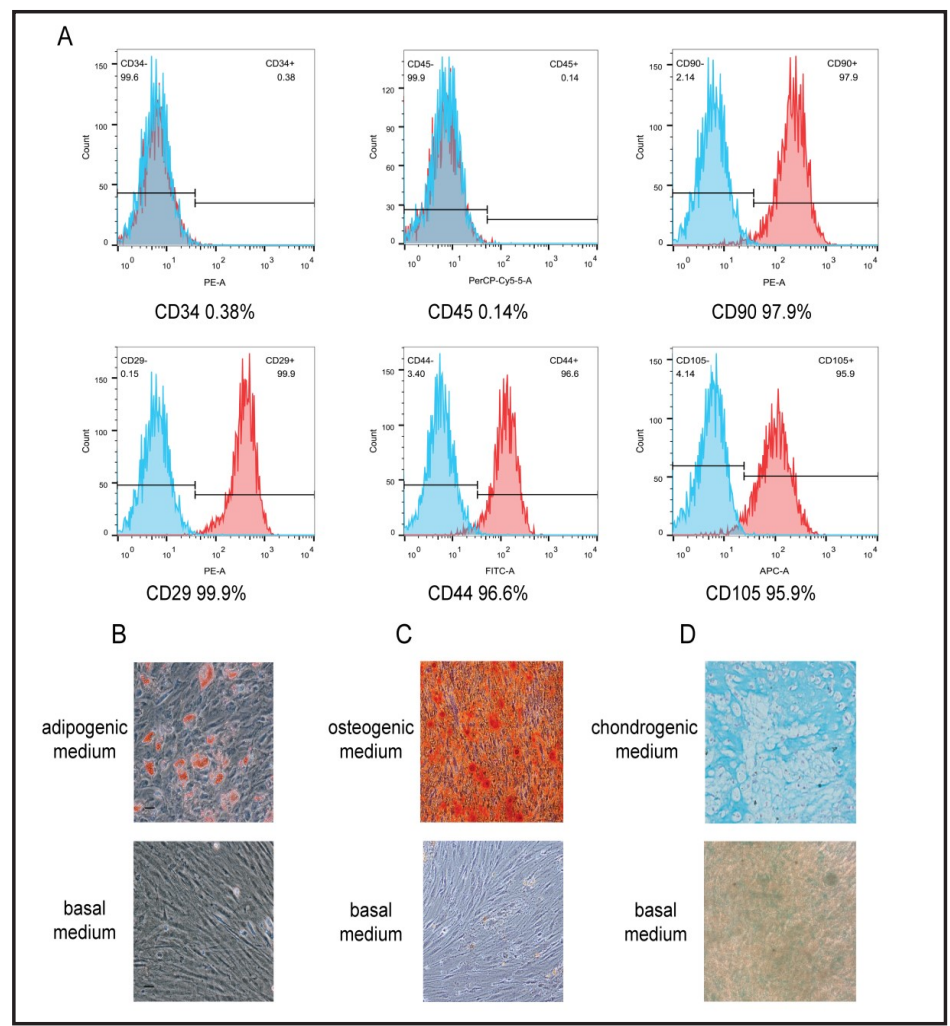

Fig. 1. Identification and characterization of hBMSCs. (A) Expression levels of hBMSC markers (CD29, CD44, CD90, CD105), a leukocyte marker (CD45), and a hematopoietic stem cell marker (CD34) in hBMSCs. (B) Adipogenic differentiation potential of hBMSCs in vitro. Oil red 0 staining of cells was detected after 14 days of culture with adipogenic medium or basal medium (BM). (C) Alizarin red staining of hBMSCs detected after 21 days of culture with osteogenic medium or BM. (D) Chondrogenic differentiation potential of hBMSC micromass in vitro. Alcian blue staining of cells was detected after 21 days of culture with chondrogenic medium or BM (magnification: $\times 100$ ).
Fig. 2. mRNA expression levels of BMP inhibitors during BMP-2-induced osteogenic differentiation in hBMSCs. (A) mRNA expression levels of BMP inhibitors at the indicated time points as measured by real-time PCR. The values were normalized to GAPDH mRNA levels and converted to the fold change on day 0. Real-time PCR was repeated independently in triplicate. (B) Western blot analysis of BMPER protein expression at the indicated time points. GAPDH was used as the loading control. (C) The band intensities corresponding to BMPER were quantified, normalized to that of GAPDH, and converted to the fold change on day 21. Representative images of three repeated experiments are shown. Data are expressed as the mean $\pm \mathrm{SD}$. ${ }^{* *}, \mathrm{P}<0.01$.

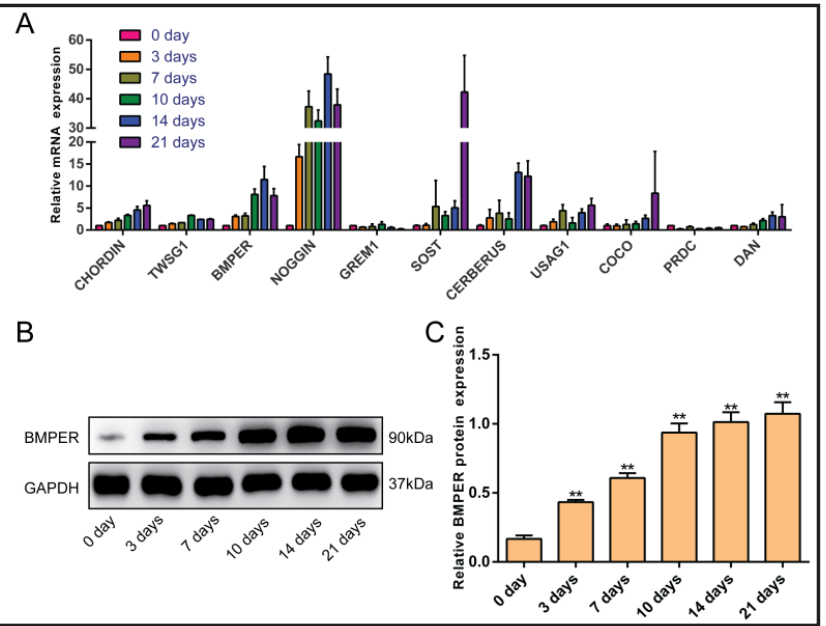




\section{Cellular Physiology Cell Physiol Biochem 2018;45:1927-1939 \begin{tabular}{ll|l} 
DOI: 10.1159/000487969 & a 2018 The Author(s). Published by S. Karger AG, Basel \\
www.karger.com/cpb
\end{tabular}

Fig. 3. BMPER positively regulates osteogenic differentiation in hBMSCs. The mRNA (A) and protein (B) expression levels of BMPER in hBMSCs infected with the pLVX-BMPER or pLVXvectors. The mRNA (C) and protein (D) expression levels of BMPER in hBMSCs transfected with BMPER negative control (NC) siRNA or BMPER siRNA. The values were normalized to GAPDH mRNA levels and converted to the fold change of the pLVX-vector or the NC-siRNA group. hBMSCs were pretreated with lentivirus, siRNA or normal control as indicated for three days, followed by treatment with basal or osteogenic media for 7 days for ALP staining (E) and the ALP activity assay (G) or 21 days for alizarin red staining (F) and the quantitative calcium assay (H). Representative images of three repeated experiments are shown. Data are expressed as the mean $\pm \mathrm{SD} . * *, \mathrm{P}<$ 0.01 .

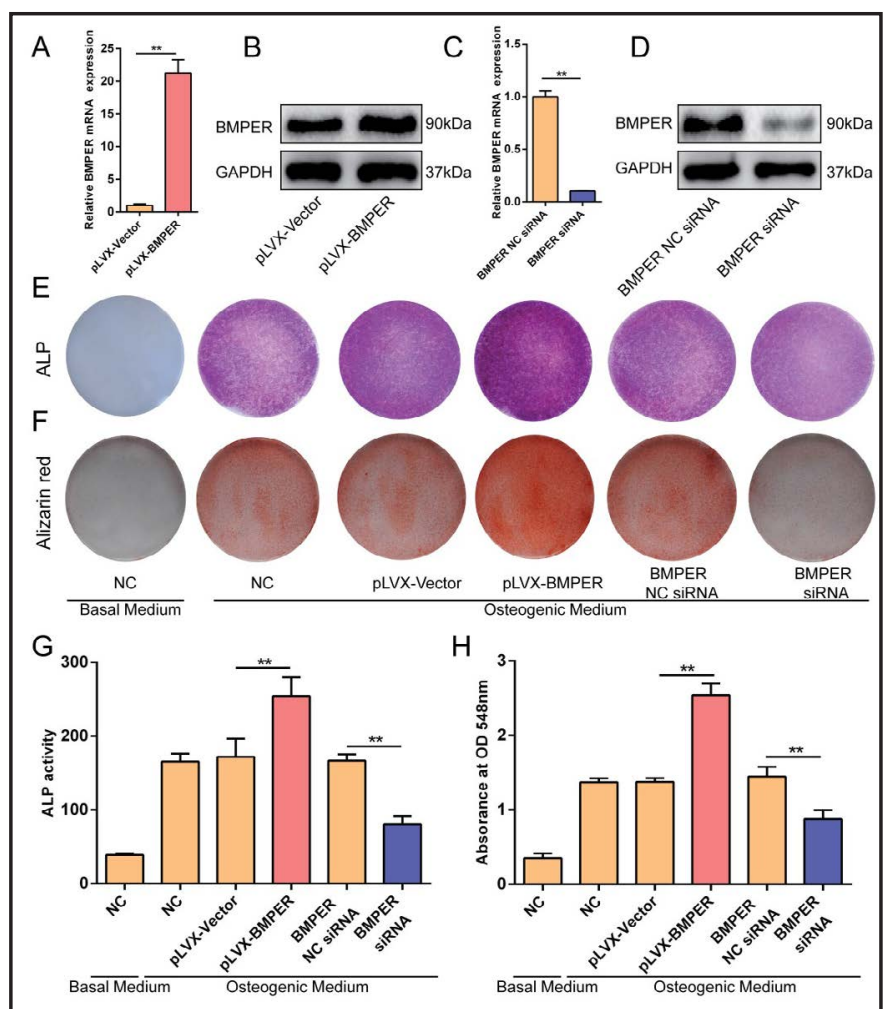

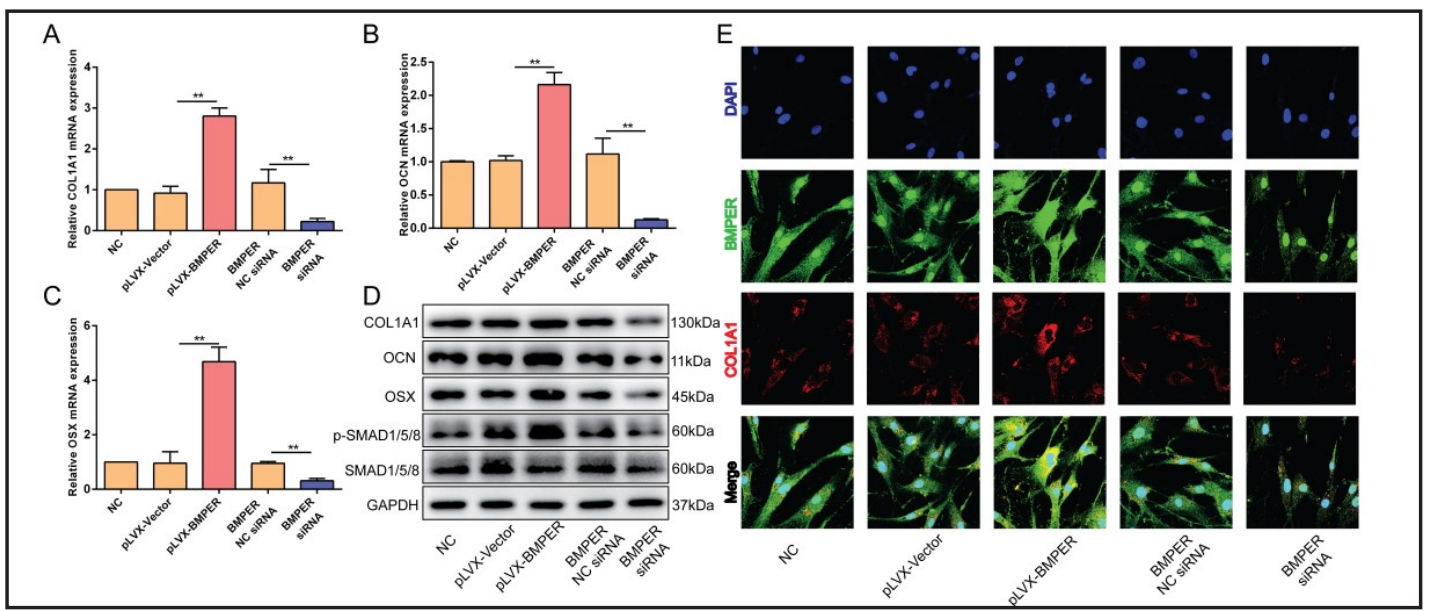

Fig. 4. BMPER promotes osteogenesis via the BMP-2/Smad signaling pathway. hBMSCs were pretreated with lentivirus, siRNA or the normal control as indicated for three days, followed by treatment with basal or osteogenic media for 7 days. Total RNA was isolated and subjected to real-time PCR using primers for the amplification of COL1A1 (A), OCN (B) and OSX (C). The values were normalized to GAPDH mRNA levels and converted to the fold change of the normal control. (D) Western blot analysis of the COL1A1, OCN, OSX, SMAD1/5/8 and p-SMAD1/5/8 proteins. GAPDH was used as the loading control. (E) COL1A1 expression as visualized by immunofluorescence analysis (magnification: $\times 400$ ). Representative images of three repeated experiments are shown. Data are expressed as the mean \pm SD. $* *, P<0.01$.

group were higher than those in the NC and pLVX-vector groups. The mRNA levels of these genes in the siRNA group were lower than those in the NC and NC-siRNA groups (Fig. 4A-C). Consistent with the mRNA expression levels, the protein expression levels of OSX, OCN, and COL1A1 were increased with BMPER overexpression and decreased with BMPER suppression (Fig. 4D). In addition, BMPER overexpression increased phosphorylated Smad1/5/8, which 


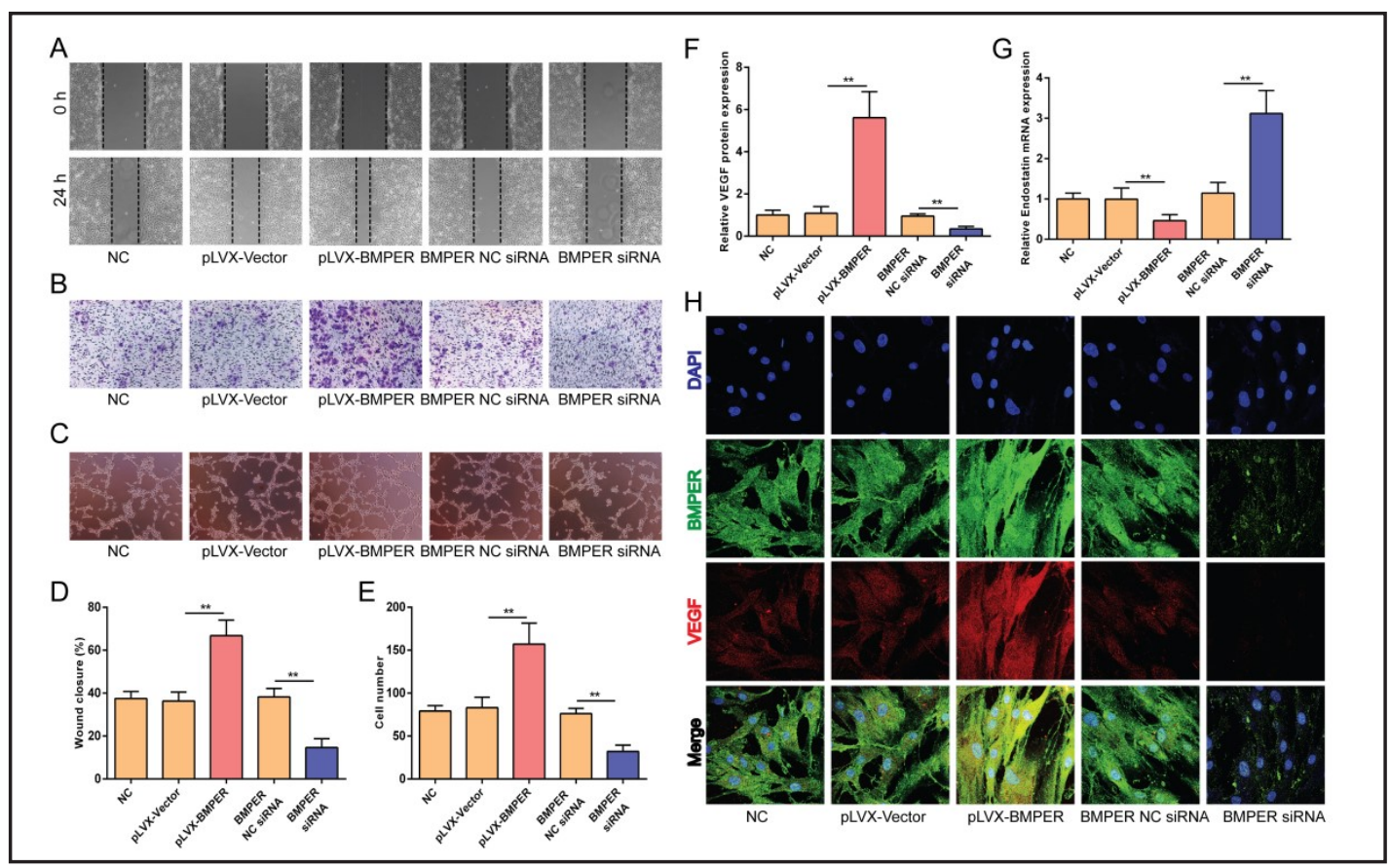

Fig. 5. Changes in BMPER in hBMSCs affect angiogenesis in vitro. hBMSCs were pretreated with lentivirus, siRNA or the normal control as indicated for three days, followed by treatment with basal or osteogenic media for 7 days, and the conditional media were collected. (A) Representative photomicrographs of wounds in HUVECs at $0 \mathrm{~h}$ and after $24 \mathrm{~h}$; the dotted lines highlight the linear scratch/wound for each group of cells. (B) HUVEC invasion abilities were determined by a Transwell migration assay. (C) Representative photomicrographs of the tube formation of HUVECs incubated with Matrigel. (D) The mean percentage of wound closure, $n=5$ per group. (E) The number of HUVECs that invaded the substratum of the membrane per view. ELISA was performed to detect the secretions of VEGF (F) and endostatin (G). (H) VEGF expression as visualized by immunofluorescence analysis (magnification: $\times 400$ ). Representative images of three repeated experiments are shown. Data are expressed as the mean $\pm \mathrm{SD}$. ${ }^{* *}, \mathrm{P}<0.01$.

is important the activation of BMP-2 signaling during osteogenesis (Fig. 4D). Furthermore, the immunofluorescence assay confirmed that COL1A1 expression increased with BMPER overexpression and decreased with BMPER suppression (Fig. 4E).

Alteration of BMPER in hBMSCs affects angiogenesis in vitro

To determine whether BMPER is involved in the osteogenesis-angiogenesis coupling process, we cultured HUVECs in conditional medium collected from osteogenic hBMSCs with altered BMPER expression. HUVECs cultured in conditional medium from the pLVX$B M P E R$ group exhibited a higher migration rate than those from the pLVX-vector group, while the siRNA-mediated downregulation of BMPER significantly weakened the migration ability of HUVECs (Fig. 5A, B, D and E). HUVECs seeded on Matrigel in conditional medium from the pLVX-BMPER group formed many branches, termed anastomosing tubes, resulting in a meshwork of capillary-like structures (Fig. 5C). By contrast, many HUVECs remained spherical and isolated when maintained in conditional medium from hBMSCs treated with BMPER siRNA, with small cellular nests and short tubes being observed (Fig. 5C). In addition, secretion of the angiogenic factor VEGF and the angiostatic factor endostatin by hBMSCs was detected. Compared with that in the pLVX-vector group, the pLVX-BMPER group showed significantly enhanced VEGF secretion and suppressed endostatin secretion (Fig. 5F and G), indicating that BMPER stimulated VEGF expression and inhibited endostatin expression. The immunofluorescence assay further confirmed that VEGF expression increased with BMPER overexpression and decreased with BMPER suppression (Fig. $5 \mathrm{H}$ ). These findings suggested that upregulated BMPER in hBMSCs enhances angiogenesis in vitro.

\section{KARGER}




\section{Cellular Physiology Cell Physiol Biochem 2018;45:1927-1939 and Biochemistry Published online: March 08, $2018 \quad$\begin{tabular}{l|l} 
DOI: 10.1159/000487969 2018 The Author(s). Published by S. Karger AG, Basel \\
www.karger.com/cpb
\end{tabular}

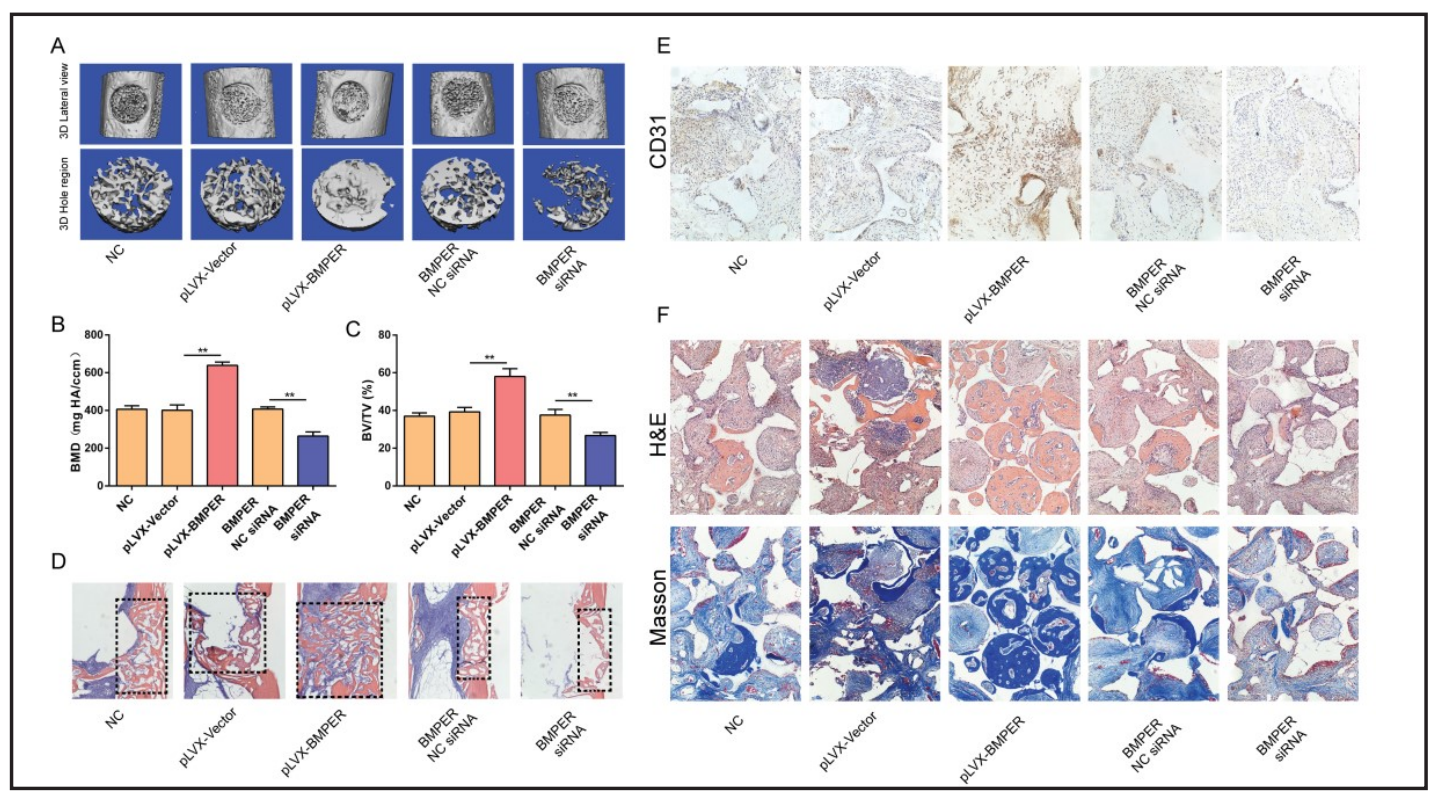

Fig. 6. Overexpression of BMPER in hBMSCs promotes angiogenesis and increases bone formation in vivo. (A) Lateral views of the 3D reconstruction of injured femoral (top panel) and mineralized bone formation in the hole region (lower panel) as determined by $\mu$ CT. Representative images from five repeated experiments in each group. (B and C) 3D structural parameters-bone mineral density (BMD) and bone volume/total volume (BV/TV) - of mineralized bone formation in the hole region as determined by $\mu C T, n=5$. (D) Representative images of HE staining showing new bone formation (magnification: $\times 100$ ). (E) Immunohistochemical staining of CD31 expression in ectopic bone masses acquired at 6 weeks (magnification: $\times 200)$. (F) Representative images of HE staining (top panel) and the corresponding Masson's Trichrome staining (lower panel) showing new bone formation (magnification: $\times 100$ ). Data are expressed as the mean $\pm \mathrm{SD}$. **, $\mathrm{P}<0.01$.

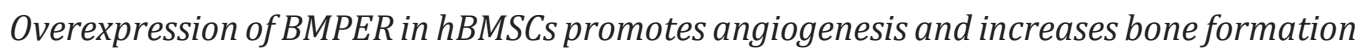
in vivo

Two animal models, the femoral monocortical defect model and the ectopic bone formation model, were established to examine the effects of BMPER on osteogenesis and angiogenesis in vivo. In the ectopic bone formation model, hBMSCs treated under the indicated condition were combined with $\beta$-TCP and then subcutaneously implanted into mice. The implants were harvested at six weeks for immunohistochemical analysis and at twelve weeks for HE and Masson's Trichrome staining. The immunohistochemical analysis confirmed that the upregulation of BMPER in hBMSCs promoted angiogenesis during ectopic bone formation, as shown by the marked increases in the numbers of CD31-positive cells and capillaries in the implants (Fig. 6E). HE and Masson's Trichrome staining confirmed that overexpression of BMPER enhanced ectopic bone formation in hBMSCs, as shown by the more robust bone formation and better mineralization (Fig. 6F). In the femoral monocortical defect model, lateral views of the 3D reconstruction of injured femurs showed more mineralized tissue in the defects of pLVX-BMPER femurs than in those of control femurs and less mineralized tissue in the defects of BMPER siRNA femurs than in those of NC femurs (Fig. 6A-C). HE staining showed that new bone covered the defective region and almost filled the bone marrow cavity in the pLVX-BMPER group, while only a small area of the hole was covered with new bone in the BMPER siRNA group (Fig. 6D). Therefore, these findings indicate that BMPER plays important roles in the osteogenic differentiation of hBMSCs and that overexpression of BMPER in hBMSCs promotes angiogenesis and increases bone formation in vivo. 


\section{Cellular Physiology Cell Physiol Biochem 2018;45:1927-1939

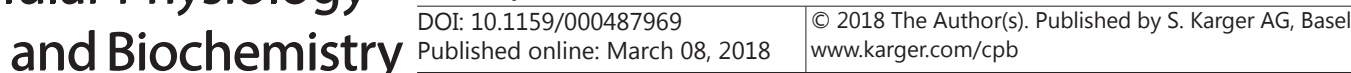 \\ Xiao et al.: BMPER Enhances Bone Formation}

\section{Discussion}

Enhancing bone formation and angiogenesis is key for the treatment of fractures, bone nonunion, delayed healing of fractures and other related diseases. BMPs play a critical role in the osteogenic differentiation of hBMSCs. However, in-depth research into the relationship between BMP inhibitors and bone formation is lacking. In this study, overexpression of one 'BMP inhibitor', BMPER, remarkably increased the BMP-2-induced osteogenic differentiation of hBMSCs both in vitro and in vivo. Furthermore, mixing $\beta$-TCP ceramic particles with hBMSCs led to extensive angiogenesis and vascularization and more mature new bone when hBMSCs overexpressed BMPER. This study extends our understanding of the role of BMPER in the osteogenesis-angiogenesis coupling process in hBMSCs, revealing that BMPER might be beneficial to human bone formation.

Most BMP inhibitors act as inhibitors by competing with BMP receptors for binding to BMP ligands $[8,9]$. Consistent with previous studies, we recently demonstrated that one BMP inhibitor, GREM2, negatively regulates the BMP-2-induced osteogenesis of hBMSCs via suppressing the BMP-2/Smad/Runx2 signaling pathway [4]. To comprehensively understand the role of other BMP inhibitors in the osteogenic differentiation of hBMSCs, we examined the mRNA expression levels of eleven BMP inhibitors during osteogenic differentiation. Although the expression of NOGGIN changed the most (Fig. 2A), we did not study this protein in depth because it plays a positive role during the osteogenic differentiation of hBMSCs [1, 12]. Numerous studies have clarified the function of $B M P E R$ in vasculature; however, little is known about the role of $B M P E R$ in osteogenesis. Since differential expression of BMPER was detected in the osteogenic differentiation process of hBMSCs (Fig. 2A-C), we propose that BMPER participates in the regulation of hBMSC differentiation. ALP and alizarin red staining confirmed that BMPER plays a positive role in the osteogenic differentiation process (Fig. 3E and F). Activation of the BMP-Smad signaling pathway is a critical process during osteogenic differentiation. When BMPs combine with BMP receptors on the BMSC membrane, Smad1/5/8 is phosphorylated and integrates with Smad4 to form a complex and is then transferred into the nucleus to promote the expression of Runx2 and other related osteogenic target genes, such as COL1A1, OCN and OSX [29]. In this study, overexpression of $B M P E R$ significantly elevated the phosphorylation of Smad1/5/8 and other related osteogenic target genes during the BMP-2-induced osteogenic process in hBMSCs (Fig. 4). Since BMP-2 remarkably promoted the expression of $B M P E R$ (Fig. 2), and upregulation of BMPER had an enhancing effect on the BMP2 signaling pathway (Fig. 4), a positive feedback mechanism accelerating osteogenic differentiation may exist. By contrast, BMPER suppression by siRNA decreases this positive feedback signal and delays the differentiation process, which may partially explain the vertebral dysplasia resulting from BMPER malfunctional mutation $[18,19]$. However, further studies are needed to reveal the specific cellular and molecular mechanisms involved in the BMPER regulation of osteogenesis.

Bone regeneration occurs in close spatial and temporal association with angiogenesis [30]. Thus, promoting vascularization by optimizing effective osteogenesis-angiogenesis coupling is essential for fracture healing [31]. In a previous study, BMPER was ascribed a pro-BMP role and shown to promote sprouting and migration in HUVECs [16]. In this study, conditional medium from hBMSCs infected with pLVX-BMPER enhanced the migration (Fig. $5 \mathrm{~A}, 5 \mathrm{~B}$ ) and network formation (Fig. 5C) abilities of HUVECs in vitro. A possible mechanism underlying this phenomenon is increased secretion of the angiogenic factor VEGF and suppressed secretion of the angiostatic factor endostatin induced by BMPER overexpression in hBMSCs during osteogenic differentiation (Fig. $5 \mathrm{~F}$ and $\mathrm{G}$ ). As a suitable anti-BMPER neutralizing antibody is lacking, we could not reveal the precise mechanism underlying the enhancing effect of the conditional medium on angiogenesis, and whether BMPER directly acts on ECs, indirectly affects ECs by promoting VEGF secretion, or exerts both effects remains unclear. CD31 is a cell adhesion molecule that is mainly expressed on the cell membranes of both developing and mature vascular ECs and is considered a marker of vascular ECs [32]. Our data showed that upregulation of BMPER in hBMSCs markedly increased the numbers 
of CD31-positive cells and capillaries in ectopic osteogenesis implants (Fig. 6E). Combined with our in vitro experiments, there is a possibility that BMPER overexpression stimulates hBMSCs to secrete more VEGF and less endostatin, thus affecting angiogenesis. However, we cannot exclude the possibility that modified hBMSCs communicate with ECs and their progenitors by other means. The mechanism underlying how the osteogenesis-angiogenesis coupling process is promoted by BMPER overexpression merits further investigation.

\section{Conclusion}

Our study demonstrates that BMPER functions as a positive regulator of osteogenesis. Importantly, our results show that upregulating BMPER enhances bone formation by promoting the osteogenesis-angiogenesis coupling process in hBMSCs, suggesting a novel therapeutic role of BMPER in the regenerative capacity of bone repair.

\section{Acknowledgements}

This work was supported by grants from the Ministry of Science and Technology of China (No. 2015DFG32200), National Natural Science Foundation of China (No.81772347, 81572123), Science and Technology Commission of Shanghai Municipality (No. 16430723500), Shanghai Municipal Education Commission-Gaofeng Clinical Medicine Grant Support (No.20161314), Shanghai Shen Kang hospital development center (No.16CR2036B) and China Postdoctoral Science Foundation (No.2017M621501).

\section{Disclosure Statement}

The authors declare no Disclosure Statement.

\section{References}

1 Chen C, Uludag H, Wang Z, Jiang H: Noggin suppression decreases BMP-2-induced osteogenesis of human bone marrow-derived mesenchymal stem cells in vitro. J Cell Biochem 2012;113:3672-3680.

-2 Canalis E, Economides AN, Gazzerro E: Bone morphogenetic proteins, their antagonists, and the skeleton. Endocr Rev 2003;24:218-235.

3 Chen Q, Shou P, Zheng C, Jiang M, Cao G, Yang Q, Cao J, Xie N, Velletri T, Zhang X, Xu C, Zhang L, Yang H, Hou J, Wang Y, Shi Y: Fate decision of mesenchymal stem cells: adipocytes or osteoblasts? Cell Death Differ 2016;23:1128-1139.

4 Wang CL, Xiao F, Wang CD, Zhu JF, Shen C, Zuo B, Wang H, Li, Wang XY, Feng WJ, Li ZK, Hu GL, Zhang X, Chen XD: Gremlin2 Suppression Increases the BMP-2-Induced Osteogenesis of Human Bone Marrow-Derived Mesenchymal Stem Cells Via the BMP-2/Smad/Runx2 Signaling Pathway. J Cell Biochem 2017;118:286297.

5 Hu K, Sun H, Gui B, Sui C: Gremlin-1 suppression increases BMP-2-induced osteogenesis of human mesenchymal stem cells. Mol Med Rep 2017;15:2186-2194.

-6 Axelrad TW, Einhorn TA: Bone morphogenetic proteins in orthopaedic surgery. Cytokine Growth Factor Rev 2009;20:481-488.

7 Hinsenkamp M, Collard JF: Growth factors in orthopaedic surgery: demineralized bone matrix versus recombinant bone morphogenetic proteins. Int Orthop 2015;39:137-147.

-8 Walsh DW, Godson C, Brazil DP, Martin F: Extracellular BMP-antagonist regulation in development and disease: tied up in knots. Trends Cell Biol 2010;20:244-256.

-9 Rosen V: BMP and BMP inhibitors in bone. Ann N Y Acad Sci 2006;1068:19-25.

10 Wan DC, Pomerantz JH, Brunet LJ, Kim JB, Chou YF, Wu BM, Harland R, Blau HM, Longaker MT: Noggin suppression enhances in vitro osteogenesis and accelerates in vivo bone formation. J Biol Chem 2007;282:26450-26459.

11 Cui ZK, Sun JA, Baljon JJ, Fan J, Kim S, Wu BM, Aghaloo T, Lee M: Simultaneous delivery of hydrophobic small molecules and siRNA using Sterosomes to direct mesenchymal stem cell differentiation for bone repair. Acta Biomater 2017;58:214-224. 


\section{Cellular Physiology Cell Physiol Biochem 2018;45:1927-1939 \begin{tabular}{l|l} 
and Biochemistry POI: 10.1159/000487969 & $\begin{array}{l}\text { C) } 2018 \text { The Author(s). Published by S. Karger AG, Basel } \\
\text { www.karger.com/cpb }\end{array}$ \\
\hline
\end{tabular}}

Xiao et al.: BMPER Enhances Bone Formation

12 Rifas L: The role of noggin in human mesenchymal stem cell differentiation. J Cell Biochem 2007;100:824-834.

13 Serpe M, Umulis D, Ralston A, Chen J, Olson DJ, Avanesov A, Othmer H, O'Connor MB, Blair SS: The BMPbinding protein Crossveinless 2 is a short-range, concentration-dependent, biphasic modulator of BMP signaling in Drosophila. Dev Cell 2008;14:940-953.

14 Kelley R, Ren R, Pi X, Wu Y, Moreno I, Willis M, Moser M, Ross M, Podkowa M, Attisano L, Patterson C: A concentration-dependent endocytic trap and sink mechanism converts Bmper from an activator to an inhibitor of Bmp signaling. J Cell Biol 2009;184:597-609.

-15 Moser M, Binder O, Wu Y, Aitsebaomo J, Ren R, Bode C, Bautch VL, Conlon FL, Patterson C: BMPER, a novel endothelial cell precursor-derived protein, antagonizes bone morphogenetic protein signaling and endothelial cell differentiation. Mol Cell Biol 2003;23:5664-5679.

-16 Heinke J, Wehofsits L, Zhou Q, Zoeller C, Baar KM, Helbing T, Laib A, Augustin H, Bode C, Patterson C, Moser M: BMPER is an endothelial cell regulator and controls bone morphogenetic protein-4-dependent angiogenesis. Circ Res 2008;103:804-812.

17 Ikeya M, Kawada M, Kiyonari H, Sasai N, Nakao K, Furuta Y, Sasai Y: Essential pro-Bmp roles of crossveinless 2 in mouse organogenesis. Development 2006;133:4463-4473.

18 Kuchinskaya E, Grigelioniene G, Hammarsjo A, Lee HR, Hogberg L, Grigelionis G, Kim OH, Nishimura G, Cho TJ: Extending the phenotype of BMPER-related skeletal dysplasias to ischiospinal dysostosis. Orphanet J Rare Dis 2016;11:1.

19 Ben-Neriah Z, Michaelson-Cohen R, Inbar-Feigenberg M, Nadjari M, Zeligson S, Shaag A, Zenvirt S, Elpeleg O, Levy-Lahad E: A deleterious founder mutation in the BMPER gene causes diaphanospondylodysostosis (DSD). Am J Med Genet A 2011;155a:2801-2806.

20 Laschke MW, Schank TE, Scheuer C, Kleer S, Shadmanov T, Eglin D, Alini M, Menger MD: In vitro osteogenic differentiation of adipose-derived mesenchymal stem cell spheroids impairs their in vivo vascularization capacity inside implanted porous polyurethane scaffolds. Acta Biomater 2014;10:4226-4235.

21 Bronckaers A, Hilkens P, Martens W, Gervois P, Ratajczak J, Struys T, Lambrichts I: Mesenchymal stem/ stromal cells as a pharmacological and therapeutic approach to accelerate angiogenesis. Pharmacol Ther 2014;143:181-196.

22 Kusumbe AP, Ramasamy SK, Adams RH: Coupling of angiogenesis and osteogenesis by a specific vessel subtype in bone. Nature 2014;507:323-328.

23 Araldi E, Schipani E: Hypoxia, HIFs and bone development. Bone 2010;47:190-196.

24 Walzer SM, Cetin E, Grubl-Barabas R, Sulzbacher I, Rueger B, Girsch W, Toegel S, Windhager R, Fischer MB: Vascularization of primary and secondary ossification centres in the human growth plate. BMC Dev Biol 2014;14:36.

25 Yao Y, Jumabay M, Ly A, Radparvar M, Wang AH, Abdmaulen R, Bostrom KI: Crossveinless 2 regulates bone morphogenetic protein 9 in human and mouse vascular endothelium. Blood 2012;119:5037-5047.

26 Xu J, Wang Y, Li J, Zhang X, Geng Y, Huang Y, Dai K, Zhang X: IL-12p40 impairs mesenchymal stem cellmediated bone regeneration via CD4+ T cells. Cell Death Differ 2016;23:1941-1951.

-27 Liao J, Wei Q, Zou Y, Fan J, Song D, Cui J, Zhang W, Zhu Y, Ma C, Hu X, Qu X, Chen L, Yu X, Zhang Z, Wang C, Zhao C, Zeng Z, Zhang R, Yan S, Wu T, Wu X, Shu Y, Lei J, Li Y, Luu HH, Lee MJ, Reid RR, Ameer GA, Wolf JM, He TC, Huang W: Notch Signaling Augments BMP9-Induced Bone Formation by Promoting the OsteogenesisAngiogenesis Coupling Process in Mesenchymal Stem Cells (MSCs). Cell Physiol Biochem 2017;41:19051923.

28 Hu K, Olsen BR: Osteoblast-derived VEGF regulates osteoblast differentiation and bone formation during bone repair. J Clin Invest 2016;126:509-526.

29 Wang C, Wang J, Li J, Hu G, Shan S, Li Q Zhang X: KDM5A controls bone morphogenic protein 2-induced osteogenic differentiation of bone mesenchymal stem cells during osteoporosis. Cell Death Dis 2016;7:e2335.

-30 Huang B, Wang W, Li Q Wang Z, Yan B, Zhang Z, Wang L, Huang M, Jia C, Lu J, Liu S, Chen H, Li M, Cai D, Jiang Y, Jin D, Bai X: Osteoblasts secrete Cxcl9 to regulate angiogenesis in bone. Nat Commun 2016;7:13885.

-31 Li J, Zhang Y, Zhao Q Wang J, He X: MicroRNA-10a Influences Osteoblast Differentiation and Angiogenesis by Regulating beta-Catenin Expression. Cell Physiol Biochem 2015;37:2194-2208.

-32 Xu DP, Zou DZ, Qiu HL, Wu HL: Traditional Chinese Medicine ShenZhuGuanXin Granules Mitigate Cardiac Dysfunction and Promote Myocardium Angiogenesis in Myocardial Infarction Rats by Upregulating PECAM-1/CD31 and VEGF Expression. Evid Based Complement Alternat Med 2017;2017:5261729. 